\title{
Constructing Tensor Network Influence Functionals for General Quantum Dynamics
}

\author{
Erika Ye \\ Division of Engineering and Applied Sciences, California Institute of Technology, Pasadena, USA 91125 \\ Garnet Kin-Lic Chan \\ Division of Chemistry and Chemical Engineering, California Institute of Technology, Pasadena, USA 91125
}

(Dated: February 12, 2021)

\begin{abstract}
We describe an iterative formalism to compute influence functionals that describe the general quantum dynamics of a subsystem beyond the assumption of linear coupling to a quadratic bath. We use a space-time tensor network representation of the influence functional and investigate its approximability in terms of the bond dimensions and time-like entanglement in the tensor network description. We study two numerical models, the spin-boson model and a model of interacting hard-core bosons in a 1D harmonic trap. We find that the influence functional and the intermediates involved in its construction can be efficiently approximated by low bond dimension tensor networks in certain dynamical regimes, which allows the quantum dynamics to be accurately computed for longer times than with direct time evolution methods. However, as one iteratively integrates out the bath, the correlations in the influence functional can first increase before decreasing, indicating that the final compressibility of the influence functional is achieved via non-trivial cancellation.
\end{abstract}

\section{INTRODUCTION}

Obtaining the long-time dynamics of a large quantum system is in general intractable due to the exponential scaling of Hilbert space with respect to system size and the associated exponential growth of spatial entanglement with time. Fortunately, in many cases, one is most interested in the dynamics of observables defined on a small subset of the full system, and can thus reframe the dynamics of the observable from the viewpoint of a subsystem coupled to a bath [1]. If one is able to determine the dynamics of the bath and its influence on the subsystem, then the original dynamics problem is reduced to obtaining the dynamics of the subsystem [2-5].

The influence functional (IF) [2] method provides an exact framework for computing the dynamics of an arbitrary bath and its interactions with the subsystem. However, the cost of computing the IF without approximation is comparable to determining the dynamics of the full system and the size of the IF scales exponentially with the number of time steps. Thus it is not usually possible to use the IF method without additional approximations.

The IF can be viewed as reweighting the path integral of the subsystem and most progress has been made for harmonic baths with linear coupling, for which Feynman and Vernon derived an analytical form of the weight [6] 7], which takes the form of the Boltzmann weight of a complex valued Hamiltonian defined in the time direction with pairwise interactions between time points.

For many physical bath spectral densities, it is natural to assume that the pairwise time interaction is shortranged in time, corresponding to a finite "memory" in the influence of the bath, and many numerical approximations have successfully taken advantage of this shortrange temporal nature [8]-21]. For IF methods, the assumption of limited memory allows one to remove the exponential growth of cost of the quantum dynamics with simulation time, thus making long time-scale quantum dynamical simulations possible. For example, in QUAPI [8], one approximates the analytical IF by only including terms acting on time steps within a finite time window. Alternatively one can construct an ansatz for the IF; a natural choice is a matrix product state (MPS) in the time direction, which compactly encodes short-range time correlations. This approach has been used in a number of recent works, which, although they do not necessarily use the language of IFs, all proceed by constructing a compressed version of the IF [22] or a closely related object such as the auxiliary density operator as defined in QUAPI [23, 24], the process tensor [25, 26], or other variants [27--30].

In this work, we are interested in using the IF method for the dynamics of a general quantum subsystem. Such a subsystem may arise as part of a larger interacting problem (in which case, the subsystem may not be different from other parts of the system) or it may arise from a system-bath model. In either case, the couplings and bath cannot be assumed to be linear and quadratic respectively and thus the analytical form of the IF is not known. Instead, the IF is simply a particular integral of the space-time dynamics that must be obtained numerically. To do this concretely, we can use a tensor network description of the space-time dynamics. For a 1dimensional representation of the system and bath, the tensor network is thus defined in $1+1$ dimensions, and the IF corresponds to a contraction of the network to yield a final matrix product state IF defined along the time direction. This procedure is similar to that presented by Banuls et al. [27, 28] and Lerose et al. [22], assuming a translationally invariant infinite problem. We will describe a general procedure to construct the IF without such assumptions and explore the numerical feasibility of doing so to compute quantum dynamics in different regimes beyond linear coupling to quadratic baths. 
The paper is organized as follows. We first translate the IF into a space-time tensor network language and describe an iterative algorithm to compute it. We then investigate the compressibility of the IF and its ability to produce long-time dynamics, first for the canonical spinboson model where the analytical IF is known, and then an interacting hard-core boson model where there is no analytical expression, which corresponds to the case of general quantum dynamics. We analyze the time-like entanglement both in the IF itself as well as the intermediates that arise as the bath is numerically integrated out. We end with a brief discussion of the implications of this work for future studies.

\section{THEORY}

\section{A. Definition of the Influence Functional}

To introduce notation, we first recall the definition of the influence functional (IF) [2]. The influence functional describes how the path integral of a subsystem is reweighted, under the influence of dynamical coupling to a bath. To obtain an explicit form, we define a full system as composed of the subsystem of interest and the coupled bath. At time $T$, we denote the subsystem density matrix by $\rho_{s}\left(s_{T}\right)$, where $s_{T}$ is a basis for the density matrix, and the bath density matrix is analogously written as $\rho_{b}\left(b_{T}\right)$. The basis of the full system is spanned by the product space $\{s\} \otimes\{b\}$. The evolution of the density matrix is given by a linear operator, the Liouville operator $L$, which we partition as $L=L_{s}+L_{b s}$ where $L_{s}$ contains the component operating only on the subsystem and $L_{b s}$ contains the component on the bath and interactions between the subsystem and bath. If we further assume the system dynamics obeys Hamiltonian evolution, then the Liouville action can be written as $L \cdot=[H, \cdot]$.

Formally, $\rho_{s}\left(s_{T}\right)$ is obtained by time evolving the entire system and tracing out the bath degrees of freedom. The path integral expression, assuming a second-order Trotter decomposition of the time evolution operator into $N$ timesteps of length $\epsilon$, is

$$
\begin{aligned}
\rho_{s}\left(s_{T}\right) & = \\
\operatorname{Tr}_{b_{T}} & {\left[\sum_{s_{t_{N-1}}} \sum_{b_{t_{N-1}}} \cdots \sum_{s_{t_{0}}} \sum_{b_{t_{0}}}\right.} \\
& \left\langle\left\langle s_{T}\left|e^{-\frac{i}{2} L_{s} \varepsilon} e^{-i L_{b s} \varepsilon} e^{-i L_{s} \varepsilon}\right| s_{t_{N-1}}, b_{t_{N-1}}\right\rangle\right\rangle \times \\
& \left\langle\left\langle s_{t_{N-1}}, b_{t_{N-1}}\left|e^{-i L_{b s} \varepsilon} e^{-i L_{s} \varepsilon}\right| s_{t_{N-2}}, b_{t_{N-2}}\right\rangle\right\rangle \times \ldots \\
\times & \left\langle\left\langle s_{t_{1}}, b_{t_{1}}\left|e^{-i L_{b s} \varepsilon} e^{-\frac{i}{2} L_{s} \varepsilon}\right| s_{t_{0}}, b_{t_{0}}\right\rangle\right\rangle \times \\
& \left.\left.\left.\left\langle\left\langle s_{t_{0}}, b_{t_{0}}\right| \rho\left(s_{t_{0}}, b_{t_{0}}\right)\right)\right\rangle\right\rangle\right]
\end{aligned}
$$

where $\rho\left(s_{t_{0}}, b_{t_{0}}\right)$ is the initial state of the system, and the double bra/ket notation indicates we are working with $\mathrm{Li}$ ouville space vectors. For simplicity, we assume there are no correlations between the subsystem and bath initially such that $\left.\left.\left.\left|\rho\left(s_{t_{0}}, b_{t_{0}}\right)\right\rangle\right\rangle=\left|\rho_{s}\left(s_{t_{0}}\right)\right\rangle\right\rangle\left|\rho_{b}\left(b_{t_{0}}\right)\right\rangle\right\rangle$. Furthermore, $L_{b s}$ is typically assumed to be diagonal in the basis $\{s\}$ (we lift both these restrictions below). Then Eq. 1 becomes

$$
\begin{aligned}
\rho_{s}\left(s_{T}\right) & =\sum_{s_{t_{N-1}}} \cdots \sum_{s_{t_{0}}}\left\langle\left\langle s_{T}\left|e^{-\frac{i}{2} L_{s} \varepsilon}\right| s_{t_{N-1}}\right\rangle\right\rangle \times \\
& \left\langle\left\langle s_{t_{N-1}}\left|e^{-i L_{s} \varepsilon}\right| s_{t_{N-1}}\right\rangle\right\rangle \times \ldots \\
& \times\left\langle\left\langle s_{t_{1}}\left|e^{-\frac{i}{2} L_{s} \varepsilon}\right| s_{t_{0}}\right\rangle\right\rangle\left\langle\left\langle s_{t_{0}} \mid \rho_{s}\left(s_{t_{0}}\right)\right\rangle\right\rangle \\
& \times I\left(s_{t_{1}}, s_{t_{2}}, \ldots, s_{t_{N}}\right)
\end{aligned}
$$

where $I\left(s_{t_{1}}, s_{t_{2}}, \ldots, s_{t_{N}}\right)$ is the influence functional

$$
\left.I(\ldots)=\operatorname{Tr}_{b_{T}}\left[e^{-i L_{b s}\left(s_{t_{N}}\right) \varepsilon} \ldots e^{-i L_{b s}\left(s_{t_{1}}\right) \varepsilon}\left|\rho_{b}\left(b_{t_{0}}\right)\right\rangle\right\rangle\right]
$$

with $L_{b s}(s)=\left\langle\left\langle s\left|L_{b s}\right| s\right\rangle\right\rangle$. The IF assigns a complex weight to each configuration of the system path integral. Consequently, the storage of the IF grows exponentially with number of time steps $N$.

\section{B. Generalized Influence Functional in Tensor Network Language}

\section{Influence Functional Structure}

Translating Eq. 1 into the tensor network language is straightforward, as shown in diagram Fig. 1(a). Similar representations have been discussed in Refs. [25. 29]. The matrix elements of $\left\langle\left\langle s_{t}\left|e^{-i L_{s} \epsilon}\right| s_{t^{\prime}}\right\rangle\right\rangle$, and $\left\langle\left\langle s_{t}, b_{t}\left|e^{-i L_{b s} \epsilon}\right| s_{t^{\prime}}, b_{t^{\prime}}\right\rangle\right\rangle$ appear as boxes with two and four legs respectively, labelled by the bra and ket basis states. The influence functional element $I\left(s_{t_{1}}, s_{t_{2}}, \ldots, s_{t_{N}}\right)$ is the object within the blue rectangle, after contraction over all bath legs. Within this diagrammatic picture it is easy to depict the generalization of the influence functional to a correlated initial state. In this case, the dotted line indicates a correlated initial state with entanglement between the subsystem and bath,

$$
\rho\left(s_{t_{0}}, b_{t_{0}}\right)=\sum_{\alpha} \rho_{s \alpha}\left(s_{t_{0}}\right) \rho_{b \alpha}\left(b_{t_{0}}\right)
$$

and the influence functional is defined with an additional index, $I\left(s_{t_{0}}, s_{t_{1}}, \ldots, s_{t_{N-1}} ; \alpha\right)$. Similarly, if $L_{b s}$ cannot be diagonalized in the subsystem basis $\{s\}$ then we can generalize the influence functional to contain two subsystem indices at each intermediate time, $I\left(s_{t_{1}} s_{t_{1}^{\prime}}, s_{t_{2}} s_{t_{2}^{\prime}}, \ldots, s_{t_{N}} s_{t_{N}^{\prime}}\right)$ as shown in Fig. 1 1 a). Given the influence functional, arbitrary time-correlation functions can be computed as shown in Fig. 1 b). 
Because the influence functional has a onedimensional structure along the time axis, it is natural to rewrite it as a matrix product state of $N$ tensors (see Fig. 1.(c)), i.e.

$$
\begin{aligned}
& I\left(s_{t_{1}}, s_{t_{2}}, \ldots, s_{t_{N}}\right)= \\
& \qquad \sum_{\{i\}} A_{i_{1}}^{(1)}\left(s_{t_{1}}\right) A_{i_{1}, i_{2}}^{(2)}\left(s_{t_{2}}\right) \ldots A_{i_{N-1}}^{(N)}\left(s_{t_{N}}\right)
\end{aligned}
$$

where $A\left(s_{t}\right)$ denotes a matrix of dimension $D \times D$, and $A\left(s_{t_{1}}\right)$ and $A\left(s_{t_{N}}\right)$ are $D$ dimensional row and column vectors respectively. In the MPS language, $D$ is referred to as the virtual bond dimension. Although any IF can be represented as a MPS for sufficiently large $D$, the MPS of small bond dimension naturally capture sums of exponentially decaying time-correlations along the time axis. The key system-specific questions to understand are thus (i) is the IF itself representable by a MPS of low bond dimension, in physically relevant dynamical and interaction regimes, and (ii) can the IF be constructed with manageable cost in those regimes. It is important to note that an affirmative answer to (i) does not imply an affirmative answer to (ii).

\section{Space-time tensor network representation}

To define an approximate procedure to construct the influence functional for complex bath dynamics, we first write down a space-time representation of the full system dynamics. We first assume that the bath Hilbert space is a product space over $K$ modes,

$$
\{b\}=\left\{b^{1}\right\} \otimes\left\{b^{1}\right\} \otimes \ldots \otimes\left\{b^{K}\right\}
$$

We can then formally express the system density matrix at any time as a matrix product state

$$
\rho\left(s_{t}, b_{t}\right)=\sum_{\{i\}} C_{i_{0}}^{(0)}\left(s_{t}\right) C_{i_{0}, i_{1}}^{(1)}\left(b^{1}\right) \ldots C_{i_{K-1}}^{(K)}\left(b^{K}\right)
$$

with a bond dimension denoted $D_{\rho}$.

Similarly, the Liouville evolution operator can be written as a matrix product operator

$$
\begin{aligned}
& \left\langle\left\langle s_{t}, b_{t}\left|e^{-i L \epsilon}\right| s_{t^{\prime}}, b_{t^{\prime}}\right\rangle\right\rangle= \\
& \quad \sum_{\{i\}} M_{i_{0}}^{(0)}\left(s_{t}, s_{t^{\prime}}\right) M_{i_{0}, i_{1}}^{(1)}\left(b_{t}^{1}, b_{t^{\prime}}^{1}\right) \ldots M_{i_{K-1}}^{(K)}\left(b_{t}^{K}, b_{t^{\prime}}^{K}\right)
\end{aligned}
$$

with a bond dimension $D_{L}$. Note that since the Liouville operator is assumed time-independent, $D_{L}$ is fixed.

The full time evolution of the system with $K$ bath modes and $N$ time steps thus corresponds to the twodimensional tensor network diagram shown in Fig. 22a). Correspondingly, the space-time representation of the influence functional is shown in Fig. 2.b).

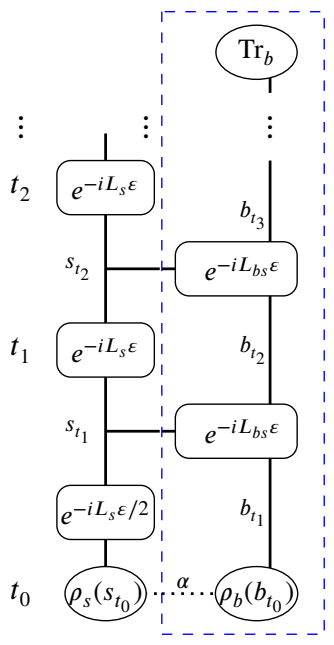

(a)

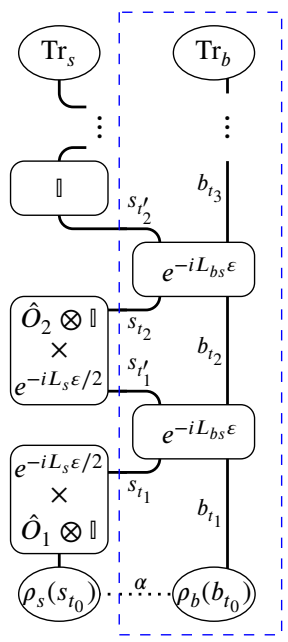

(c)

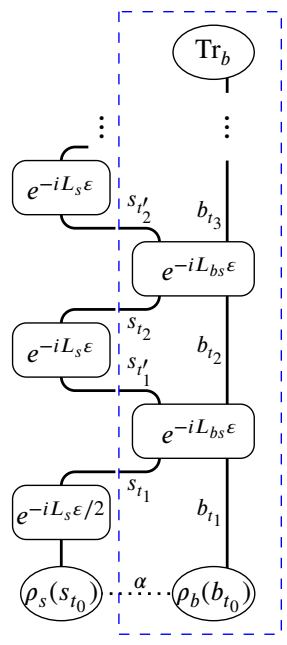

(b)

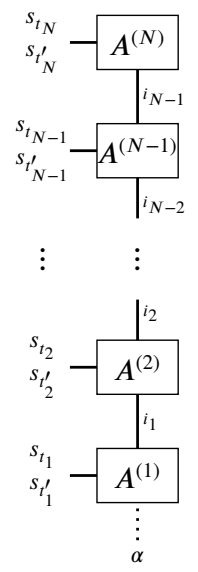

(d)
FIG. 1. (a, b) Time evolution of $\rho\left(s_{t_{0}}\right)=\sum_{\alpha} \rho_{s, \alpha}\left(s_{t_{0}}\right) \otimes \rho_{b, \alpha}\left(s_{t_{0}}\right)$ in Liouville space with second order Trotter decomposition between system and interaction dynamics. The boxed regions are the traditional and generalized definitions of the influence functional, respectively. (c) Measurement of the time-correlation $\left\langle\hat{O}_{2}\left(t_{1}\right) \hat{O}_{1}\left(t_{0}\right)\right\rangle_{\rho}$. (d) Matrix product state representation of influence functional. The labels $\left\{s_{t_{m}}\right\}$ and $\left\{b_{t_{m}}\right\}$ index the system and bath states at time step $m$, respectively. The labels $\left\{i_{m}\right\}$ index the virtual bonds. Lines that connect two tensors (blocks) represent tensor contraction over the labeled indices.

\section{Transverse Contraction Scheme}

The most common way to contract a $2 \mathrm{D}$ space-time tensor network is from bottom to top, i.e. in the direction of increasing time. [31-38]. We refer to this as direct time evolution. For example, contracting the network in Fig. 2 a) row by row yields the system density matrix at each time step as an MPS. For exact evolution $D_{\rho}$ must grow by a factor of $D_{L}$ at each time step. This 


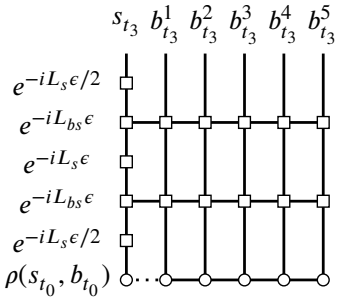

(a) time evolution $\mathrm{TN}$

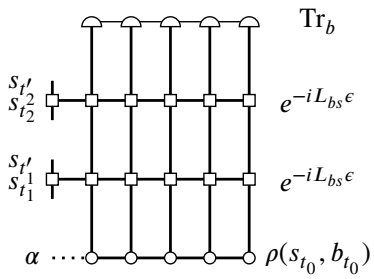

(b) influence functional TN
FIG. 2. (a) Space-time tensor network representing time evolution of a system represented as a 1D MPS (row of circles) with second order Trotter decomposition between system and interaction dynamics. Pictured are two time steps applied to a system coupled to five bath sites. (b) Tensor network representation of influence functional containing only 2 time steps. The semicircles represent the trace operation, which can be written as a MPS of bond dimension 1 .

means that in the worst case, $D_{\rho}$ grows exponentially with time. The cost of such a time evolution time step is then $\mathcal{O}\left(D_{\rho}^{3} K N d_{\rho}\right)$ with $D_{\rho} \sim e^{\lambda N}$ to maintain fidelity during time evolution, and $d_{\rho}$ the dimension of the density matrices represented at each MPS site.

However, if the time-correlations in the influence functional decay with long time, then this implies that the influence functional ultimately can be represented by a matrix product with low bond dimension along the time axis. This suggests that a more efficient contraction strategy is to contract column wise (in the transverse direction to time). The cost of constructing the final influence functional is thus $\mathcal{O}\left(D_{I}^{3} K N D_{L}\right)$. $D_{I}$ will grow by $d_{\rho}$ during contraction, but it need not have a dependence on the total simulation time.

In practice, exact contraction of the $2 \mathrm{D}$ tensor network (in either direction) is often too expensive. In Banuls et al [27, 28], the explicit transverse contraction of the $2 \mathrm{D}$ tensor network was avoided by assuming that the system is infinite and translationally invariant, in which case the result of the infinite contraction of columns is proportional to the maximal eigenvector of the column transfer operator.

Alternatively, one may use standard matrix product state techniques to compress the intermediates that arise during the contraction to restrict bond dimensions $D_{\rho}$ or $D_{I}$ to some constant value [31-33]. In this paper, we use such an approximate transverse contraction scheme (compressing to a fixed bond dimension of the contraction intermediates) to obtain the IF (Fig. 2. b)) for systems with arbitrary baths. The algorithm involves iteratively contracting and compressing the columns from the edges of the bath inwards to the sites connected to the subsystem (Fig. 3). Assuming the subsystem is defined as the leftmost site, we start from the rightmost boundary column, and then the column is absorbed leftward to make a new boundary column, which is compressed using stan- dard MPS compression to a pre-specified maximum bond dimension $D_{I}$, following the "boundary contraction" algorithm of 2D tensor networks [39]. In the hardcore boson calculations where there is no special subsystem and the bath can extend both to the left and right of the site of interest, we first "fold" the tensor network so that both left and right baths lie to one side of the subsystem (see Appendix). All tensor networks have redundant degrees of freedom (a gauge) and the choice of this gauge affects the quality of the compression. We choose the gauge as shown in Fig. 3 This contraction scheme was implemented using quimb [40].

\section{Numerical time evolution and initial state preparation}

To construct the influence functional tensor network we require a matrix product representation of the time evolution operator $e^{-i L_{b s} \epsilon}$. If the Hamiltonian consists of only nearest neighbor interactions, one can rewrite the operator as a Trotter-Suzuki decomposition of nearest neighbour gates which we then directly map onto a matrix product operator. Otherwise, for more general interactions one can use a 4th-order Runge-Kutta expansion [41]. The resulting matrix product operator can then have large bond dimension, but we compress it down to a truncation error of $\mathcal{O}\left(\epsilon^{5}\right)$. In the cases studied here, the subsystem is small enough such that $e^{-i L_{s} \epsilon}$ can be obtained exactly.

For purposes of comparison, we will also present reference dynamics generated by standard MPS time evolution (ie. contracting the space-time tensor network in the usual time direction) [31, 33, 41, 42]. Because the underlying full system dynamics is governed by Hamiltonian evolution in the problems that we study, we have the option to apply $e^{-i H \epsilon}$ as a commutator to the square root of the density matrix $\left(\left[e^{-i H \epsilon} \rho^{1 / 2}\right]\left[\rho^{1 / 2 \dagger} e^{i H \epsilon}\right]\right)$ or via $e^{-i L \epsilon}$ directly. We refer to the former as Hilbert time evolution (HTE) and the latter as Liouville time evolution (LTE). In HTE, the compressed tensor network dynamics is carried out for the pseudowavefunction $\psi=e^{-i H \epsilon} \rho^{1 / 2}$ [43-45]. HTE has the advantage that the compressed density matrix is always positive definite, although correlations between the bra and ket sides of the density matrix are less compressible. In existing literature, this is sometimes referred to as purification-based time evolution. In the case that $\rho$ is a pure state, this method is equivalent to traditional MPS Hilbert space time evolution.

\section{RESULTS}

\section{A. Spin Boson Model}

First, we consider the well-studied spin-boson model, in which a single spin is linearly coupled to a bath of non- 


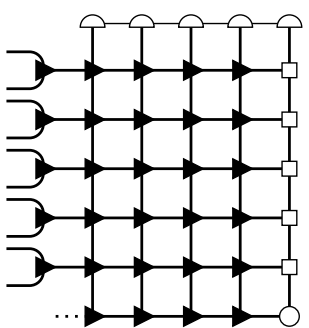

initialize

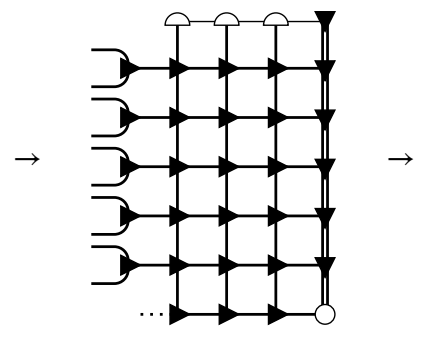

contract + canonicalize
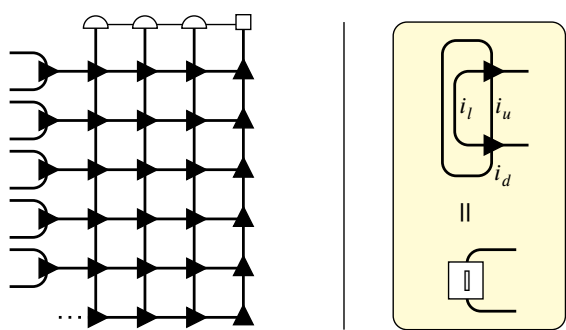

compress

FIG. 3. Transverse contraction iteration of IF tensor network. The time evolution operators $e^{-i L_{b s} \epsilon}$ at each time step are the rows of the grid and are each represented as an MPO, and the subsystem of interest is at the left-most site. Before contraction, we first convert each $e^{-i L_{b s} \epsilon}$ MPO into left canonical form [33. 46] as indicated by the right pointing arrows along the rows. The rightmost two columns are then contracted and compressed to fixed bond dimension $D_{I}$ using the standard MPS compression algorithm, where the column is first converted into a canonical form (here, top canonical form) and then compressed by singular value decomposition in the reverse direction (leaving it in bottom canonical form) [33]. The canonical form implies that the tensors satisfy an isometric condition (diagram on the right); e.g. the right pointing arrow implies contraction of a tensor with its complex conjugate over the left, up, and down indices yields the identity matrix. The procedure is repeated until all columns have been contracted.

interacting harmonic oscillators,

$H_{S B}=\Delta S_{X}+\int d \omega\left[S_{Z}\left(g(\omega) a_{\omega}+g(\omega)^{*} a_{\omega}^{\dagger}\right)+\omega a_{\omega}^{\dagger} a_{\omega}\right]$

where $\Delta$ is the tunneling strength between the two subsystem states, and the system-bath coupling strength $g(\omega)$ is determined from the bath spectral density function $J(\omega)$ by

$$
|g(\omega)|^{2}=\frac{1}{\pi} J(\omega)
$$

In the case of an Ohmic bath with exponential cut-off,

$$
J(\omega)=\frac{\pi}{2} \alpha \omega e^{-\omega / \omega_{c}}
$$

where $\alpha$ is the Kondo parameter and $\omega_{c}$ is the cut-off frequency. Typically one computes the dynamics from a factorized initial state $\left.\left.\left|\rho_{s}\left(s_{t_{0}}\right)\right\rangle\right\rangle\left|\rho_{b}(\beta)\right\rangle\right\rangle$, where $\left.\left|\rho_{b}(\beta)\right\rangle\right\rangle$ is the Gibbs thermal state of the isolated bath at finite temperature $\beta$. In this paper, we set $\Delta=1.0, \omega_{c}=7.5$, and $\beta=5.0$.

The spin-boson model exhibits a dynamical phase transition from thermalizing to localizing behavior at $\alpha=$ $1.0+\mathcal{O}\left(\Delta / \omega_{c}\right)$ [23] , and is often cited as an example of physically relevant non-Markovian dynamics [1, 47]. Because of the linear coupling and harmonic bath, the IF may be computed via an analytical expression. There already exist several methods of obtaining accurate dynamics for various bath coupling strengths and spectral densities [23, 48]. We thus use this model as a benchmark to understand the properties of the influence functional, its compressibility, and the accuracy of the tensor network contraction approximation.

\section{Compressibility of Analytical IF}

We first investigate the compressibility of the analytical expression for the influence functional for the spinboson model. Denoting the system basis $|s\rangle\rangle \equiv\left|s^{+}\right\rangle\left\langle s^{-}\right|$ where $\left|s^{ \pm}\right\rangle \in\{|1\rangle,|-1\rangle\}$ and $|1\rangle,|-1\rangle$ are the eigenstates of the $S_{Z}$ operator, then the influence functional can be written as

$$
I_{S B}=\exp \left\{-\sum_{k=1}^{N} \sum_{k^{\prime}=1}^{k}\left(s_{t_{k}}^{+}-s_{t_{k}}^{-}\right)\left(\eta_{k k^{\prime}} s_{t_{k^{\prime}}}^{+}-\eta_{k k^{\prime}}^{*} s_{t_{k^{\prime}}}^{-}\right)\right\}
$$

This explicitly shows the form of the influence functional as the Boltzmann weight of a complex spin Hamiltonian with the spins interacting along the time axis via the long-range pairwise "interaction" $\eta_{k k^{\prime}}$. We can further factorize the weights into contributions for times $\left(t_{1}\right)$, $\left(t_{1}, t_{2}\right), \ldots,\left(t_{1}, t_{N}\right)$, giving

$$
\begin{aligned}
I_{S B}= & \prod_{k=1}^{N} I_{0}\left(s_{t_{k}}^{ \pm}\right) \prod_{k=1}^{N-1} I_{1}\left(s_{t_{k}}^{ \pm}, s_{t_{k+1}}^{ \pm}\right) \ldots \\
& \prod_{k=1}^{N-m} I_{m}\left(s_{t_{k}}^{ \pm}, s_{t_{k+m}}^{ \pm}\right) \cdots \prod_{k=1}^{1} I_{N}\left(s_{t_{k}}^{ \pm}, s_{t_{k+N-1}}^{ \pm}\right)
\end{aligned}
$$

where

$$
I_{m}=\exp \left\{-\left(s_{t_{k+m}}^{+}-s_{t_{k+m}}^{-}\right)\left(\eta_{k+m, k} s_{t_{k}}^{+}-\eta_{k+m, k} s_{t_{k}}^{-}\right)\right\}
$$

The $\eta_{k k^{\prime}}$ interaction terms can be derived from the spectral density of the bosonic bath. Expressions for $\eta_{k k^{\prime}}$ are given in Eq. (12) of Ref. 8 , where they use $\Delta t$ instead of $\epsilon$ to denote the timestep.

In QUAPI, a finite memory approximation is made by only considering interactions $\eta_{k k^{\prime}}$ for times $k, k^{\prime}$ within 
$m_{\max }$ of each other [8]. This approximation is only valid if $\eta_{k, k+m}$ decays quickly with respect to $m$, which is the case for many smooth spectral densities. Given this approximation, one can evaluate the influence functional with a cost exponential in $m_{\max }$. Under similar assumptions, one also expects the matrix product state representation of the IF (Eq. 5) to be compressible to small bond dimension. One way to verify this would be to construct the large influence functional object as an exact tensor, and then compress it into a matrix product state. Because of the exponential storage of the tensor with time, this is possible only for a small number of time points $N$. Alternatively, one could build the influence functional iteratively (i.e. piece by piece in Eq. 13) and compress at each step. This is the idea behind TEMPO and related methods [23, 24] which exploit the compressibility of the augmented density matrix, the influence functional applied to the subsystem density matrix, i.e.

$$
A\left(s_{t_{1}}, \ldots, s_{t_{m_{\max }}}\right)=\sum_{s_{t_{0}}} I\left(s_{t_{0}}, \ldots, s_{t_{m_{\max }}}\right) \rho_{s}\left(s_{t_{0}}\right)
$$

Note that because $I$ is composed of commuting pieces there are many possible decompositions and thus sequences of iterative constructions.

To verify the compressibility of the IF itself, we approximate $I_{S B}$ as an MPS of bond dimension $D_{I}$ using an iterative scheme (see Appendix) and determine the error in the resulting on-site dynamics, using the $D_{I}=128$ result as reference. Here and throughout the paper, the error is computed as the r.m.s. deviation of the dynamics of an observable with respect to some reference over the time interval of the plot. The results are shown in Fig. 4 We find that the analytical influence functional is relatively compressible, regardless of coupling strength. Even at a small bond dimension of $D_{I}=16$, the correct behavior of the dynamics in both the thermalization and localization regimes is captured [23, 48]. Compared to results obtained using $m_{\max }=8$, the MPS algorithm yields slightly more accurate dynamics near the localization transition. The key advantage of using a compressed matrix representation, as opposed to only truncating $\eta_{k, k+m}$ at some $m_{\max }$ as in QUAPI, is that this does not eliminate the effects of long-range memory [23]. Overall, this result confirms that for certain spectral densities, the influence functional can be efficiently written as a low-rank MPS.

\section{Finite Size Harmonic Bath}

We now use the spin-boson model to examine if the IF can be constructed efficiently when there is no known analytical expression. To do so, we consider a finite size harmonic bath with $K$ sites. Here, the analytical IF (for infinite boson cap) can still be computed, but because the bath is discrete we can also compute the IF by the transverse contraction algorithm, and for small baths and finite
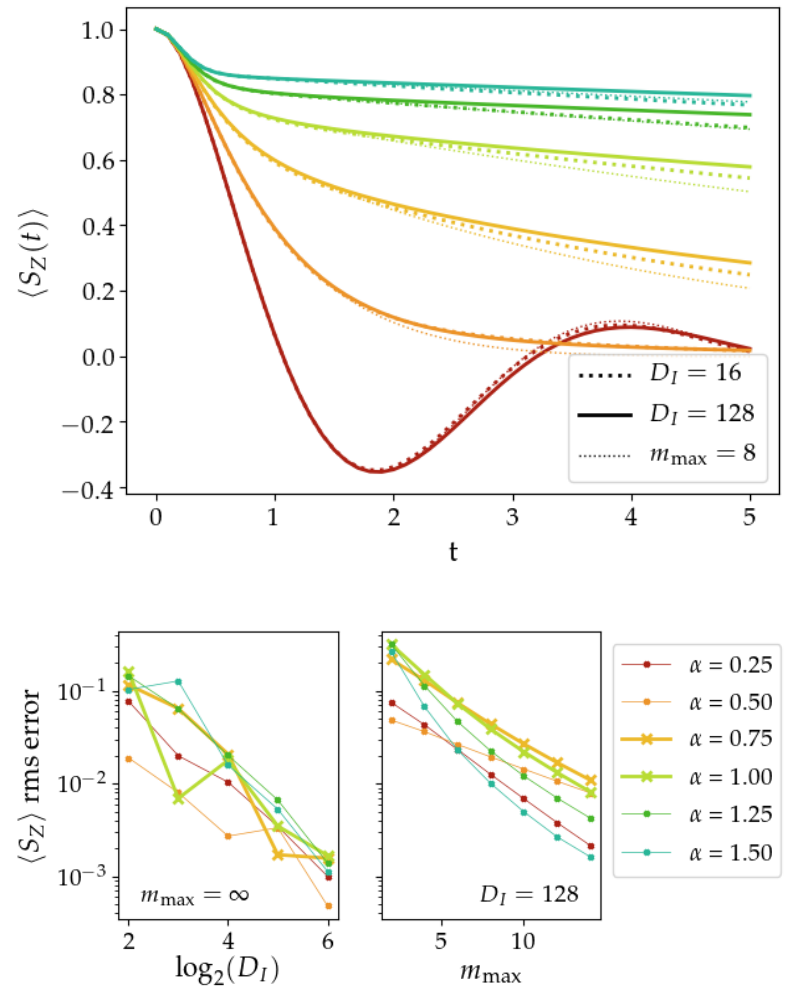

FIG. 4. (top) Dynamics obtained using the analytical IF capped to $m_{\max }$ and compressed to bond dimension $D$ where (thick dotted) $D=16, m_{\max }=\infty$, (thin dotted) $D=128, m_{\max }=8$, and (solid) $D=128, m_{\max }=\infty$, for various coupling strengths $\alpha$. (bottom, left) R.m.s. error for the analytical IF with respect to D. (bottom, right) R.m.s error for capped IFs with respect to $m_{\max }$ computed using $D=128$. The errors are obtained using dynamics from the IF with no cap $\left(m_{\max }=\infty\right)$ and $D=128$ as reference. The error of the complete IF with respect to $D$ is independent of coupling strength. In contrast, the error for finite $m_{\max }$ is larger near the localization transition. These calculations are for $N=50$ time steps of size $\varepsilon=0.1$. The system parameters are $\Delta=1.0$, bath inverse temperature $\beta=5.0$, and an Ohmic bath spectral density with exponential cut-off $\omega_{c}=7.5$.

boson cap, the transverse contraction can be performed without compression. The bath is characterized by the discretized spectral density,

$$
J_{D}(\omega)=\sum_{j} \frac{J(\omega)}{\rho(\omega)} \delta\left(\omega-\omega_{j}\right)
$$

We use a linear discretization of the bath sites, such that the bath density is $\rho(\omega)=K / \omega_{m}$, where $\omega_{m}$ is the maximum boson frequency used. Here, we set $\omega_{m}=10$.

First, we consider a system with only 2 bath modes each with a maximum boson number of 2 , for $N=100$ time steps of size $\epsilon=0.05$. For this small system, we use the exact time evolution operator of $L_{b s}$ and compute the IF by exact transverse contraction, applying compression only to the final IF object. Fig. 5. a) shows the error of the 


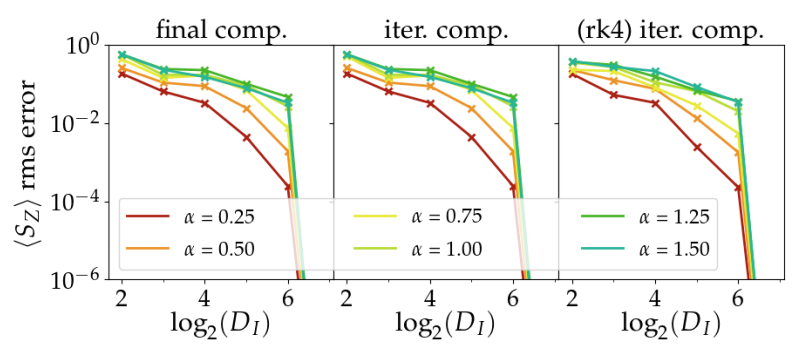

FIG. 5. Comparisons of average error in $\left\langle S_{Z}(t)\right\rangle$ with respect to reference direct time evolution results for the IF where the IF is (left) constructed using exact time evolution, contracted exactly, and finally compressed to bond dimension $D_{I}$ at the end, and where the IF is constructed with (middle) exact time evolution and (right) RK4 time evolution but iteratively contracted and compressed using the transverse compression scheme. In these calculations, we use $\Delta=1.0$, bath inverse temperature $\beta=$ 5.0, cut-off frequency $\omega_{c}=7.5$, and assume a discrete Ohmic bath with 2 modes at $\omega=[5.0,10$.$] . The bosonic bath sites$ are approximated to have only a maximum boson number of 2 . Time evolution is performed using $N=100$ time steps with a time step of $\epsilon=0.05$. The plots show that for small bath sizes, the error in the iterative compression scheme is dominated by the compressibility of the final IF.

exact IF compressed to bond dimension $D_{I}$. As expected, the IF is much less compressible than with the continuous bath density in the last section, due to the small bath size. The error decreases only slightly until it drops suddenly once the bond dimension is large enough to capture the IF exactly, and further, the compression error increases with $\alpha$. We then perform the same comparison but for IFs computed by transverse contraction with compression, for both exact and RK4 time evolution. As seen in Fig. 5 the errors are comparable to those obtained when compressing the final exact IF. This indicates that for this small problem, there is little additional error added by the iterative contraction, and that the time-step error is negligible: the error is dominated by the compressibility of the final IF itself (which is low when the bath size is small).

Next we investigate systems with larger bath sizes in Fig. 6(a). We first examine the time-dynamics of the analytical IF (i.e. without any boson cap, and without transverse contraction) for a discretized spectral density with 11 bath sites, as well as the IF computed by transverse contraction, using a boson cap of 2 . Because the system size is so small, the exact reference dynamics for a boson cap of 2 can be generated by direct MPS time evolution (here we use $D_{\rho}=64$ and Hamiltonian time evolution). From this comparison we observe two things. First, compared to using the continuous bath density, the error of the analytical IF dynamics is increased, although it is still somewhat compressible. For the same $D_{I}$, the errors using transverse contraction are larger, suggesting that at intermediate points in the transverse contraction, there is more time-like entanglement than in the final IF itself. In
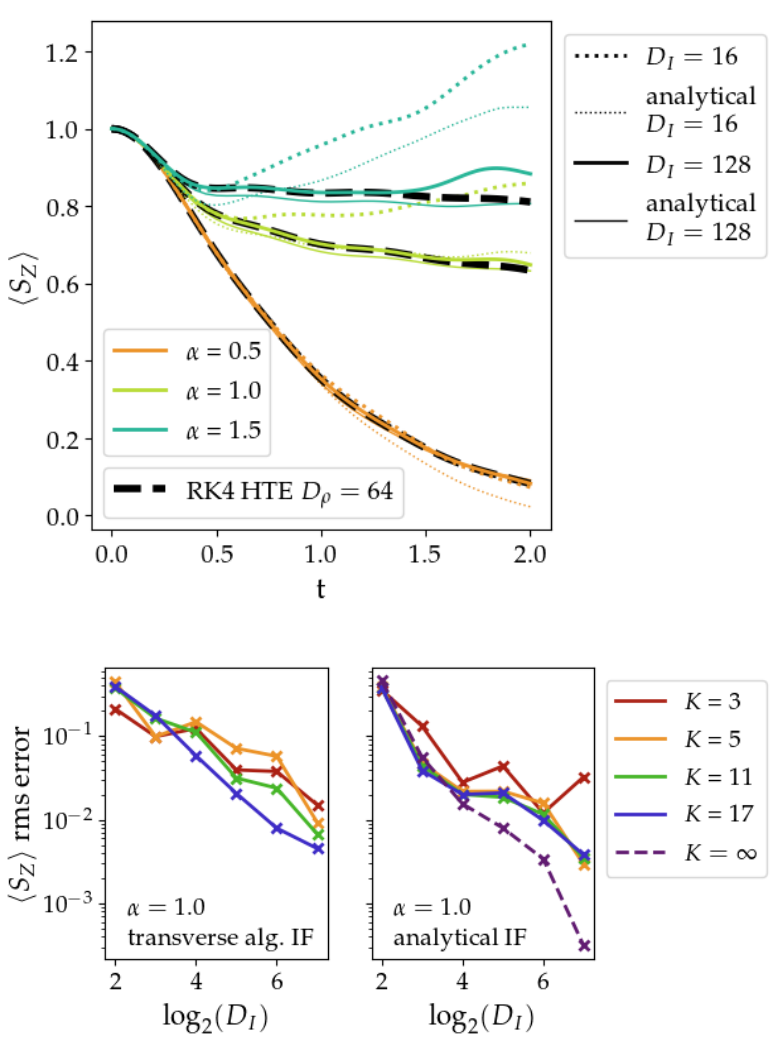

FIG. 6. (top) Expectation values $\left\langle S_{Z}(t)\right\rangle$ obtained from the analytical IF (thinner colored lines) and IF from transverse contraction (thicker colored lines) for bath size $K=11$ with $\alpha=$ $0.5,1.0$, and 1.5 . The thick dashed line corresponds to reference dynamics from direct time evolution of the density matrix. The transverse contraction scheme introduces additional error with respect to the analytical result, which increases with $\alpha$. (bottom) Time-averaged error in IF dynamics with respect to $D_{I}=128$ results, obtained using (left) the transverse contraction scheme and (right) the analytical IF for the finite bath of size $K$, respectively. The $K=\infty$ bath size corresponds to the continuous bath.

Fig. 6(b) we show the time-averaged error of the IF dynamics as a function of the number of bath sites. We see that this error decreases as the number of bath sites increases, both for the analytical IF and the transverse contraction. This is consistent with the idea that smoother bath densities are more "compressible".

\section{B. 1D Hard-core Boson Model}

We next study dynamics of 1D hard-core bosons (HCB) described by the Hamiltonian

$$
H_{H C B}=\sum_{j}\left[-J\left(a_{j}^{\dagger} a_{j+1}+\text { h.c. }\right)+U n_{j} n_{j+1}+\frac{K}{2} n_{j} j^{2}\right]
$$



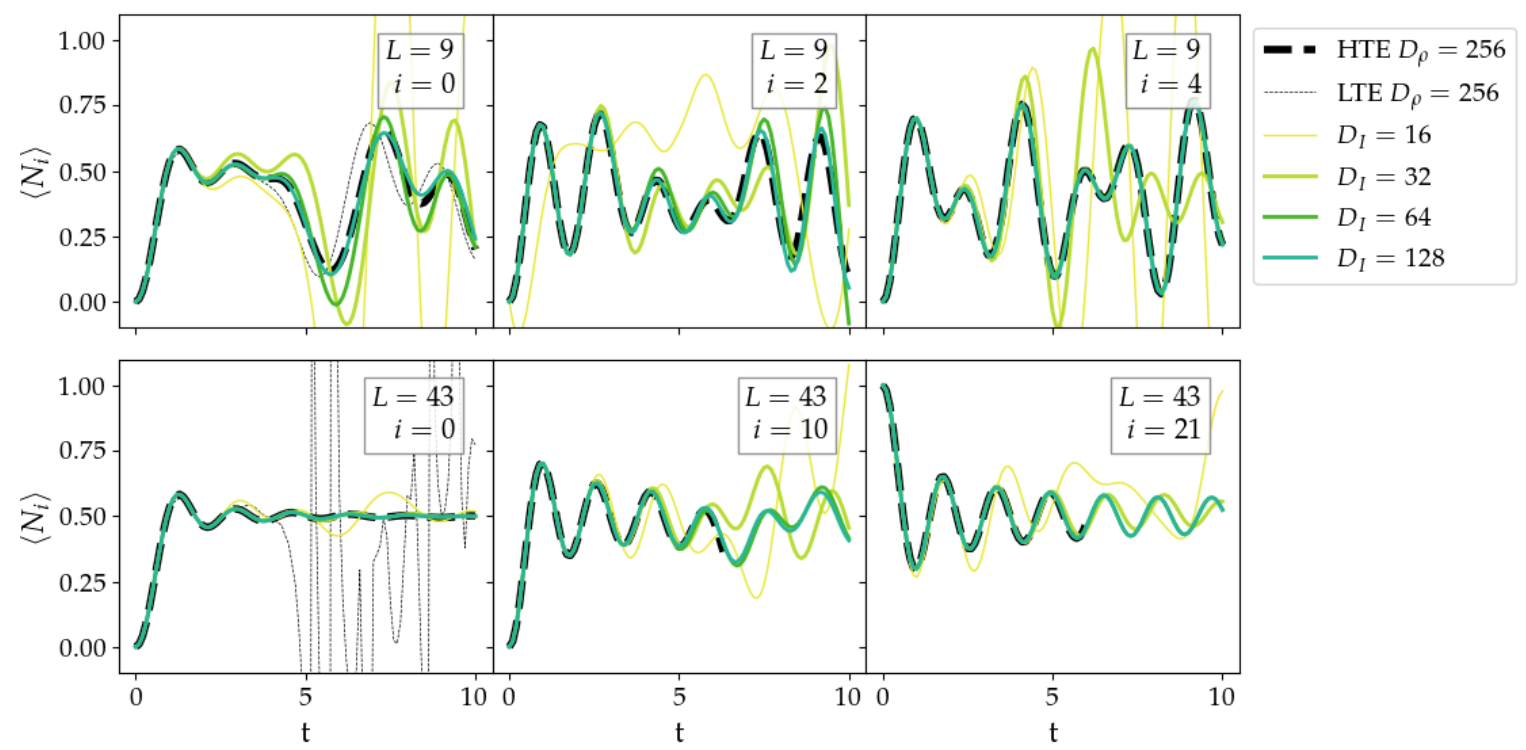

FIG. 7. Expectation values $\left\langle N_{i}(t)\right\rangle$ for the hard-core boson model with $U=0$ for system sizes (top) $L=9$ and (bottom) $L=43$ for sites $i=\{0, L / 2, L / 4\}$ obtained using the iterative contraction scheme with bond dimension $D_{I}$ as labeled. The thick dashed line is the dynamics obtained by direct Hilbert-space time evolution, compressed to bond dimension $D_{\rho}=256$, shown only up to times for which the results are converged. The thin dashed line, shown only in the left-most plots but with similar behavior for all, is the dynamics obtained by direct time evolution of the full density matrix. Unphysical behavior suggests loss of positivity of the density matrix. For these calculations, the initial state is a pure product state with alternating spins, $|0,1,0,1, \ldots\rangle$. Time evolution is performed using Trotter steps with a $N=100$ time steps of $\Delta t=0.1$. Consistent with earlier observations, larger $D_{I}$ is needed to accurately capture the IF for smaller bath sizes. However, as shown in the $L=43, i=21$ simulation, the IF of comparable bond dimension can simulate dynamics for longer times than direct HTE.

where $a_{j}^{\dagger}, a_{j}$ are hard-core boson creation and annihilation operators at the $j^{\text {th }}$ lattice site, and $n_{j}=a_{j}^{\dagger} a_{j}$. This Hamiltonian is similar to some used in cold atom dynamics, and thus we add a harmonic potential term to emulate a cold atom trap potential. We assume a pure initial state $|0,1,0, \ldots\rangle$ such that there is one particle at every other lattice site, and set the parameters $J=1$ and $K=10^{-2}$ while varying $U$. For non-zero interaction term $U$, there is no analytical form for the IF.

We compute the dynamics of $\left\langle N_{i}(t)\right\rangle$ at lattice sites $i=\{0, L / 4, L / 2\}$, where $L$ is the length of the 1-D chain. For longer chains, the rapid growth of entanglement means that direct MPS time evolution (either using Hamiltonian evolution, denoted HTE or Liouvillian evolution, denoted LTE) with a finite $D_{\rho}$ can only obtain converged dynamics up to a finite time. We consider two chain lengths: $L=9$ where converged HTE MPS dynamics can be used as a reference, and $L=43$, where the HTE MPS dynamics appear to be not fully converged (no longer within 0.03 of $D_{\rho}=512$ results) for the full simulated time. To obtain the dynamics using the influence functional method, we partition the lattice such that site $i$ is the subsystem of interest and the remaining sites are the bath.
The $U=0$ dynamics for $L=9$ and $L=43$ is shown in Fig. 7 Direct MPS LTE shows unphysical behaviour for large system sizes, presumably because of the loss of positivity at some point in the dynamics. In contrast, the dynamics obtained using the iteratively contracted IF are more stable, highlighting the innate compressibilty of time evolution tensor network along the time axis as opposed to the spatial axis. For $L=9$, the IF dynamics only appears to begin to converge by $D_{I}=128$ with respect to the (exact) HTE dynamics, having less than 0.03 r.m.s error in $\left\langle N_{i}(t)\right\rangle$ over the simulated time interval and deviations within 0.08 . Thus, there appears to be no significant advantage to using the the IF method over direct HTE for small system sizes. Conversely, for the $L=43$ system, the IF dynamics are converged with respect to $D_{I}=256$ results by $D_{I}=64$, with less than 0.01 r.m.s error and a maximum deviation of 0.04 for $i=10$ and less than 0.004 r.m.s error and a maximum deviation of 0.001 for $i=21$. Note that the $i=10$ dynamics converge more slowly because effectively the site is coupled to two separate baths, one of which is small. However, the IF method still outperforms direct HTE.

For $U>0$, as shown in Fig. 8. the $D_{I}=64$ results appear less converged than the non-interacting case, but the $D_{I}=128$ results are converged (r.m.s. errors of 

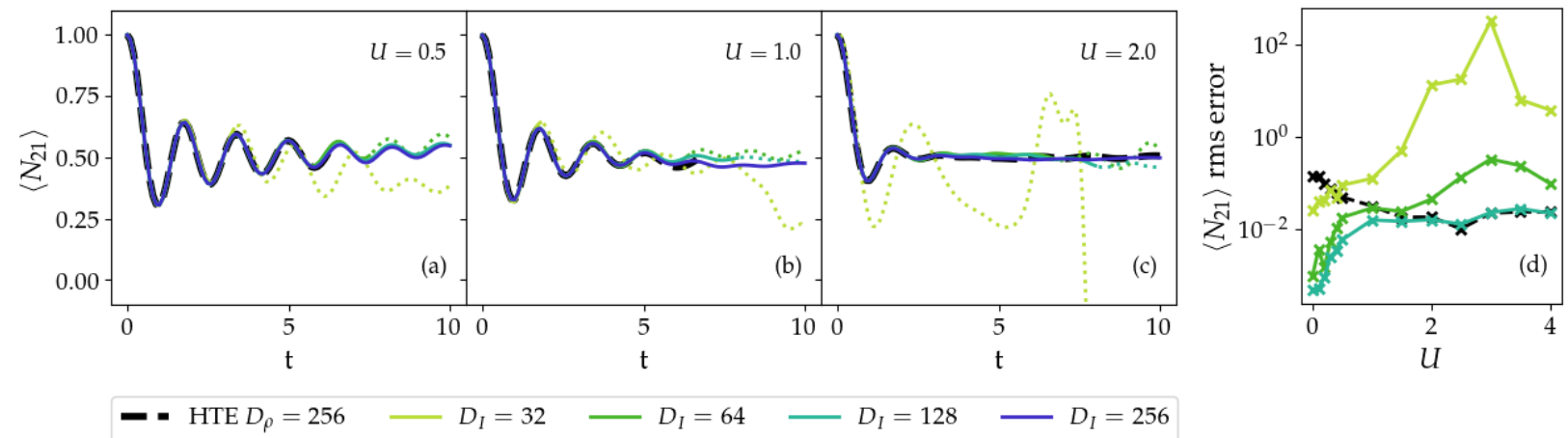

FIG. 8. (a,b,c) Expectation values of the site-occupancy $\left\langle N_{i}(t)\right\rangle$ for the hard-core boson model of length $L=43$ at lattice site $i=21$ for different coupling strengths $U=0.5,1.0$, and 2.0. Lines become dotted after divergence of $>0.03$ with respect to the $D_{I}=256$ IF results. (d) The r.m.s. errors with respect to the $D_{I}=256 \mathrm{IF}$ results as a function of $U$. Simulation parameters are otherwise the same as in Fig. 7 Compared to the $U=0$ case, a larger bond dimension is needed, particularly at around $U=3.0$ where the r.m.s. error peaks. In contrast, the HTE dynamics converge more quickly with increasing $U$.

the $D_{I}=128$ observable dynamics with respect to the $D_{I}=256$ results, for times longer than that accessible by direct time evolution, are less than 0.03 ). This shows that the IF-based dynamics can produce the correct oscillatory behaviour of the density as a function of time, which is not captured by the direct MPS time evolution despite using a larger bond dimension (this difficulty with the long time oscillatory tail has previously been noted in other cold atom simulations [49]). However, while the IF method notably outperforms direct HTE at small $U$, the two methods become comparable at larger $U \approx 1.5$ once the oscillatory tail is sufficiently dampened.

\section{Entanglement Spectrum}

The accuracy of the transverse contraction scheme depends on the entanglement in the time-like direction. Recall that our contraction algorithm starts with the farthest column (an MPS), and at each iteration another column is contracted into this boundary. Thus, as the iteration number increases, the boundary column represents more of the bath. For both the spin-boson model and HCB model, we measure the singular values at the middle of the boundary "bath" MPS during each step of the iterative contraction scheme. The entanglement entropy (EE) and spectrum of the singular values (normalized so that $\sum_{i}\left|s_{i}\right|^{2}=1$ ) are plotted in Fig. 9 For the spin-boson model, only results for $\alpha=1.0$ are shown; the only notable difference for other $\alpha$ is that the EE increases with $\alpha$. Consistent with the observations in our simulations above, the EE decreases as one increases bath size. For the SB model, the EE decreases with increased number of bath sites in the discretization until convergence. For the HCB model, only if sufficiently large enough bond dimension is used does the EE decrease with increasing iteration number. Otherwise, the EE stays at a large value throughout the contraction scheme and the gap between the dominant and non-dominant singular values decreases; this makes the EE of the smaller $D_{I}$ approximation larger than that of the larger $D_{I}$ approximation. Overall, this suggests that the final compressibility of the IF emerges from the cancellation of many different correlations as one iteratively contracts out the bath.

\section{CONCLUSIONS}

In this work, similar to some other recent contributions [22, 26], we have used the representation of the influence functional within the tensor network language, motivated by the limitations of modeling spatial entanglement growth in quantum dynamics. We have discussed a transverse tensor network contraction algorithm that allows us to compute the influence functional in cases where the analytical form is not known. We have applied this algorithm to study both the canonical spinboson model as well as an interacting hard-core boson chain where the bath is not quadratic (i.e. interacting). We find that the compressibility of the influence functional is controlled by several factors, principally the size of the bath, as well as the nature of the interactions. In addition, although the time-like correlations may ultimately be short-ranged in the final influence functional, during the transverse tensor network contraction to construct it, it is possible to proceed through intermediate quantities with larger time-like entanglement. This suggests a complicated picture where time-like correlations first accumulate as the bath is integrated out before finally cancelling in the influence functional itself. In the regimes 

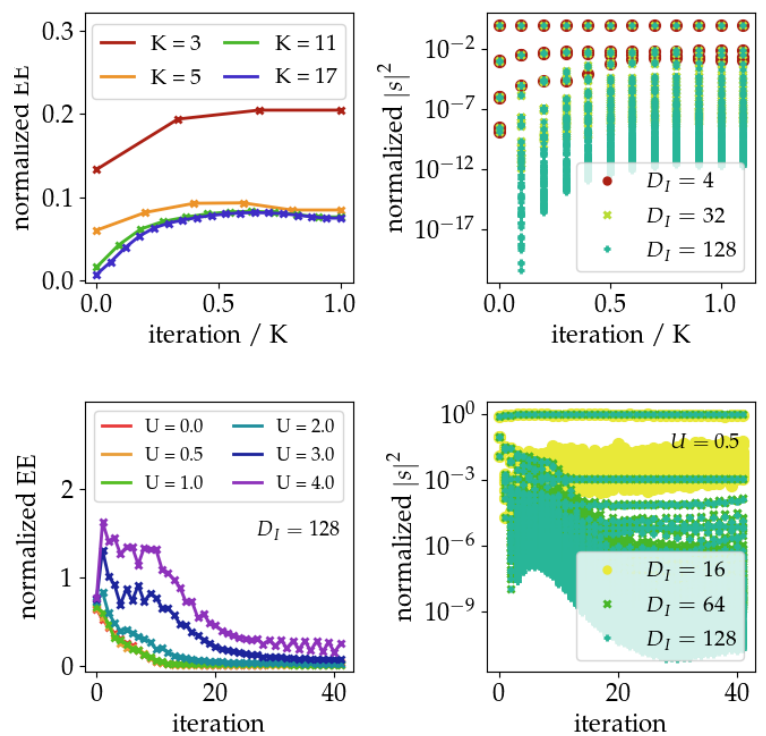

FIG. 9. (Top left) Entanglement entropy and (top right) spectrum of normalized singular values at the middle of the "bath" boundary column MPS after each contraction and compression iteration for the SB model $(\alpha=1.0)$. As bath size increases, the $\mathrm{EE}$ of the IF decreases, converging to some finite value. (Bottom left) Entanglement entropy of the hard-core boson model with $L=43$ for the "bath" boundary MPS with contractions starting from the right edge, plotted for different values of $U$. The decrease in EE with respect to iteration shows that EE decreases with system size. (Bottom right) Normalized singular values for the hardcore-boson model with $L=43$ and $U=0.5$. Surprisingly, for insufficient $D_{I}$, the singular values take on large and incorrect values, yielding an artificially large EE.

where the influence functional and all intermediate quantities are compressible, as in some interaction regimes in the interacting hardcore boson model we have studied, it is possible to outperform conventional tensor network time evolution methods at longer times.

There are many possible directions for further investigation. For example, there are natural extensions to higher-dimensional interacting problems and fermionic systems, as well as more complicated correlation functions. Also, a better theoretical understanding of how correlations grow and cancel out in the transverse contraction scheme may lead to a deeper understanding of the generation of memory in quantum dynamics, improved contraction schemes, and ultimately new algorithms to carry out longer time dynamical simulations.

\section{ACKNOWLEDGMENTS}

GKC was supported by the Center for Molecular Magnetic Quantum Materials, an Energy Frontier Research Center funded by the U.S. Department of Energy, Office of Science, Basic Energy Sciences under Award No. DE-
SC0019330. EY was primarily supported by the Google $\mathrm{PhD}$ fellowship program with supplemental salary support from the Center for Molecular Magnetic Quantum Materials.

\section{Appendix A: Matrix Product Form of Analytical IF}

As discussed in the text, the expression for the analytical IF in discretized time steps is given by Eq. 12 and we wish to write it in the MPS form with physical bonds that index the states of the density matrix at each timestep,

$$
\begin{aligned}
& I\left(s_{t_{1}}, s_{t_{2}}, \ldots, s_{t_{N}}\right)= \\
& \sum_{i_{1}, \ldots, i_{N-1}} A\left(s_{t_{1}}\right)_{i_{1}} A\left(s_{t_{2}}\right)_{i_{1}, i_{2}} \ldots A\left(s_{t_{N}}\right)_{i_{N-1}}
\end{aligned}
$$

One possible way to construct the IF is to take the product of factors in Eq. 12 in the order $I=I_{0} I_{1} \ldots I_{N-1}$. We start by using the $I_{0}$ terms which are in the form of a product state (MPS with bond dimension 1). We then multiply by each of the subsequent $I_{m}$ and compress into an MPS after each $I_{m}$ is applied. Multiplying by $I_{m}$ can be viewed as multiplication by an MPO where the tensors are very sparse. The two body terms $I_{m}$ for $m>1$ are long-range operators, and must be padded with identities to skip over the times in the middle. More concretely, the MPO for an IF with 6 time steps can be written as

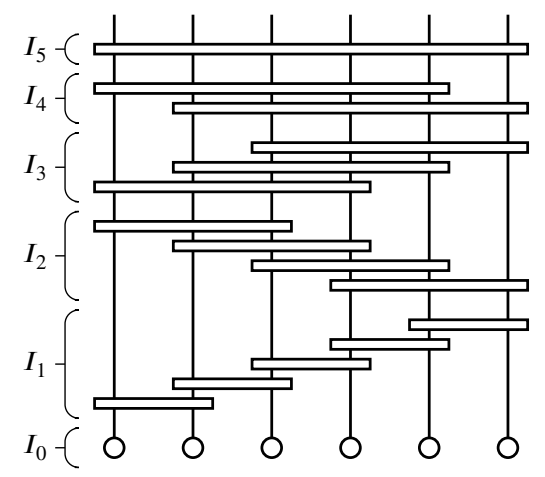

with the two body terms $I_{m}$ constructed as

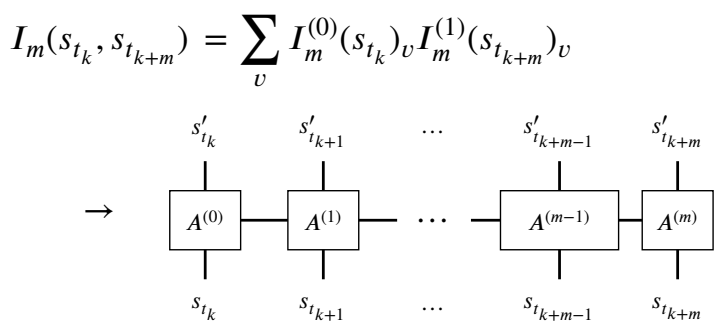


where

$$
\begin{aligned}
& A_{s_{t_{k}}^{\prime}, s_{t_{k}}, v_{0}}^{(0)}=\sum_{j}\left(\delta_{s_{t_{k}}, s_{t_{k}}^{\prime}, j}\right) I_{\left(j, v_{0}\right)}^{(0)} \\
& A_{s_{t_{k+m}}^{\prime}, s_{t_{k+m}}, v_{k-1}}^{(k)}=\sum_{j}\left(\delta_{s_{t_{k+m}}, s_{t_{k+m}}^{\prime}, j}\right) I_{\left(j, v_{k-1}\right)}^{(1)} \\
& A_{s_{t_{i}}^{\prime}, s_{t_{i}}, v_{i-1}, v_{i}}^{(i)}=\delta_{v_{i}, v_{i+1}} \delta_{s_{t_{i}}, s_{t_{i}}^{\prime}} \quad \forall i \in[1, \ldots, m-1]
\end{aligned}
$$

Note that in the first equality we decompose $I_{m}$ into two tensors, which can be done by a SVD or QR decomposition. To contract the network, we start from the bottom and work our way up. Because each $I_{m}$ factor commutes with the rest, other choices of ordering are possible and are the basis of algorithms such as TEMPO [23, 24].

\section{Appendix B: Influence functional transverse contraction around an arbitrary site}

Sometimes the site whose dynamics we are interested in may be at the middle of the MPS representation of the system (e.g. in the hard core boson model). Thus, we need to generalize the tensor network diagrams presented in the main text to consider IFs for subsystems at arbitrary lattice site $i$.

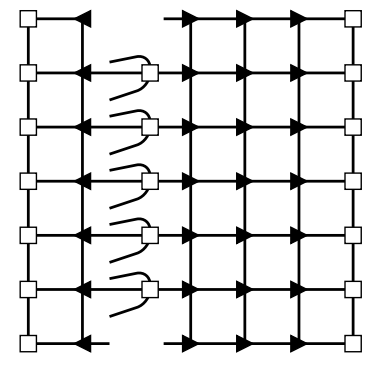

influence functional

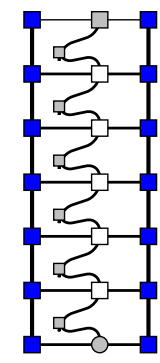

expectation value
FIG. 10. (left) Tensor network showing generalized IF isolating dynamics at the $i=3$ lattice site. Triangles denote gauging of tensors along the row, as defined in Fig. 3 in the main text. (right) Tensor network computing expectation value using [blue] left and right environment columns obtained separately using the iterative contraction scheme described above, [white] original tensors at site $i$ dictating interactions of both environment columns with site itself and the environments with each other, and [gray] on-site terms including [circle] the initial state and [square] time evolution operators and the observable of interest (see Fig. 11). It is cheapest to contract this network vertically from the row at one end and continuing to the other end. Note that in using this method one does not explicitly compute the IF itself.

If the Hamiltonian only consists of nearest-neighbor interactions, the IFs from the two sides of site $i$ are separable and can be computed independently. Otherwise, the tensor network can be initialized as shown in Fig. 10. and one contracts inwards from the outer columns separately. Once only the column corresponding to the isolated site is left, one can now include on-site terms (initial state, onsite time evolution operators, observable) such that the network now corresponds to the expectation value of the observable at the desired time step (a scalar). The cost of contracting this network scales like $\mathcal{O}\left(D_{I}^{3}\right)$, which is much cheaper than explicitly computing the full IF first and then computing the observable expectation values.
[1] H. Breuer and F. Petruccione, The theory of open quantum systems (Oxford University Press, 2002).

[2] R. P. Feynman, Rev. Mod. Phys. 20, 367 (1948)

[3] S. Nakajima, Progress Theor. Phys. 20, 948 (1958)

[4] R. Zwanzig, J. Chem. Phys. 33, 1338 (1960)

[5] H. Breuer, B. Kappler, and F. Petruccione, Ann. Phys. 291, 36 (2001)

[6] R. P. Feynman and F. L. Vernon, Annals Phys. 24, 118 (1963)

[7] F. L. Vernon, The Theory of a General Quantum System Interacting with a Linear Dissipative System, Ph.D. thesis California Institute of Technology (1959).

[8] N. Makri and D. E. Makarov, J. Chem. Phys 102, 2600 (1995)

[9] N. Makri, J. Chem. Phys. 111, 6164 (1999).

[10] A. Strathearn, B. W. Lovett, and P. Kirton, New J. Phys.
19, 093009 (2017)

[11] S. Weiss, J. Eckel, M. Thorwart, and R. Egger, Phys. Rev. B 77, 195316 (2008)

[12] D. Segal, A. J. MIllis, and D. R. Reichman, Phys. Rev. B 82, 205323 (2010).

[13] G. Cohen, E. Gull, D. R. Reichman, and A. J. Millis, Phys. Rev. Lett. 115, 266802 (2015)

[14] H.-T. Chen, G. Cohen, and D. R. Reichman, J. Chem. Phys. 146, 054105 (2017)

[15] J. Cerillo and J. Cao, Phys. Rev. Lett. 112, 110401 (2014).

[16] R. Rosenbach, J. Cerrillo, S. F. Huelga, J. Cao, and M. B. Plenio, New J. Phys. 18, 023035 (2016).

[17] Q. Shi and E. Geva, J. Chem. Phys. 119, 12063 (2003).

[18] A. Kelly, A. Montoya-Castillo, L. Wang, and T. E. Markland, J. Chem. Phys. 144, 184105 (2016).

[19] G. Cohen and E. Rabani, Phys. Rev. B 84, 075150 (2011) 
[20] L. Kidon, H. Wang, M. Thoss, and E. Rabani, J. Chem. Phys. 149, 104105 (2018).

[21] S. Chatterjee and N. Makri, J. Phys. Chem. B 123, 10470 (2019)

[22] A. Lerose, M. Sonner, and D. A. Abanin, arXiv cond-mat , 2009.10105 (2020)

[23] A. Strathearn, P. Kirton, D. Kilda, J. Keeling, and B. W. Lovett, Nature Communications 9, 3322 (2018)

[24] M. R. Jorgensen and F. A. Pollock, Phys. Rev. Lett. 123, $240602(2019)$

[25] F. A. Pollock, C. Rodriguez-Rosario, T. Frauenheim, M. Paternostro, and K. Modi, Phys. Rev. A 97, 012127 (2018)

[26] M. Cygorek, M. Cosacchi, A. Vagov, V. M. Axt, B. W. Lovett, J. Keeling, and E. M. Gauger, arXiv quant-ph, 2101.01653 (2021)

[27] M. C. Bañuls, M. B. Hastings, F. Verstraete, and J. I. Cirac, Phys. Rev. Lett. 102, 240603 (2009).

[28] A. Müller-Hermes, J. I. Cirac, and M. C. Bañuls, New J. Phys. 14, 075003 (2012)

[29] I. A. Luchnikov, S. V. Vintskevich, H. Ouerdane, and S. N. Filippov, Phys. Rev. Lett. 122, 160401 (2019)

[30] I. A. Luchnikov, S. V. Vintskevich, D. A. Grigoriev, and S. N. Filippov, Phys. Rev. Lett. 124, 140502 (2020)

[31] G. Vidal, Phys. Rev. Lett. 03, 040502 (2004)

[32] A. J. Daley, C. Kollath, U. Schollwock, and G. Vidal, J. Stat. Mech.: Theor. Exp. , P04005 (2004)

[33] U. Schollwöck, Ann. Phys. 326, 96 (2011)
[34] S. White and A. E. Feiguin, Phys. Rev. Lett. 93, 076401 (2004)

[35] C. D. White, M. Zalatel, R. S. K. Mong, and G. Refael, Phys. Rev. B 97, 035127 (2018)

[36] U. Schollwöck and S. R. White, AIP Conference Proc. 816, 155 (2006)

[37] E. Ronca, Z. Li, C. Jimenez-Hoyos, and G. K.-L. Chan, J. Chem. Theory Comput. 13, 5560 (2017)

[38] J. Haegeman, C. Lubich, I. Oseledets, B. Vandereycken, and F. Verstraete, Phys. Rev. B 94, 165116 (2016)

[39] V. Murg, F. Verstraete, and J. I. Cirac, Phys. Rev. A 75, 033605 (2007)

[40] J. Gray, Journal of Open Source Software 3, 819 (2018)

[41] J. J. García-Ripoll, New J. Phys. 8, 305 (2006)

[42] M. Zwolak and G. Vidal, Phys. Rev. Lett. 93, 207205 (2004)

[43] F. Verstraete, J. J. Garcia-Ripoll, and J. I. Cirac, Phys. Rev. Lett. 93, 207204 (2004)

[44] T. Barthel, U. Schollwöck, and S. R. WHite, Phys. Rev. B 79, 245101 (2009)

[45] C. Karrasch, J. H. Bardarson, and J. E. Moore, Phys. Rev. Lett. 108, 227206 (2012)

[46] G. Vidal, Phys. Rev. Lett. 91, 147902 (2003)

[47] I. d. Vega and D. Alonso, Rev. Mod. Phys. 89, 015001 (2017)

[48] H. Wang and M. Thoss, New J. Phys. 10, 115005 (2008)

[49] S. Trotzky, Y.-A. Chen, A. Flesch, I. P. McCulloch, U. Schollwock, J. Eisert, and I. Bloch, Nature Phys. 8, 325 (2012) 